\title{
Superimposed Mechanomyographic Response at Different Contraction Intensity in Medial Gastrocnemius and Soleus Muscles
}

\author{
Yoichi Ohta*, Norihiro Shima** and Kyonosuke Yabe* \\ *Department of Sport and Exercise Sciences, Graduate School of Sport Sciences, Osaka University of Health and Sport Sciences \\ 1-1 Asashirodai, Kumatori, Sennan-gun, Osaka 590-0496 Japan \\ 205d02@ouhs.ac.jp \\ ** National Institute of Fitness and Sports \\ Shiromizu, Kanoya, Kagosima 891-2393 Japan \\ [Received November 1, 2006 ; Accepted December 26, 2006]
}

\begin{abstract}
This study examined changes in mechanical properties of medial gastrocnemius muscle (MG) and soleus muscle (SOL) at different contraction intensities using superimposed mechanomyogram (MMG) signals. The subjects were measured for the superimposed twitch amplitude and peak acceleration of the twitch torque development $\left(\mathrm{d}^{2} T / \mathrm{d} t^{2}\right)$ from electrical stimulation of the posterior tibial nerve during isometric plantar flexions at $20 \%, 40 \%, 60 \%, 80 \%$, and $100 \%$ of maximal voluntary contraction (MVC). In addition, the superimposed MMG amplitude was recorded from MG and SOL. The superimposed twitch amplitude, $\mathbf{d}^{2} T / \mathbf{d} t^{2}$ and superimposed MMG amplitude of MG and SOL decreased with increasing \%MVC. With increasing \%MVC, changes in the pattern of superimposed MMG of the MG were similar to the changes in the pattern of $\mathbf{d}^{2} T / \mathbf{d} t^{2}$. There were significant relationships between the superimposed twitch amplitude and the superimposed MMG amplitude of MG and SOL, and the $\mathrm{d}^{2} T / \mathrm{d} t^{2}$ and superimposed MMG amplitude of MG and SOL. The normalized superimposed MMG amplitude of the MG at $20 \%$ and $40 \%$ of MVC is significantly higher than that of SOL. These results suggest that superimposed MMG signals recorded from synergistic muscle (MG and SOL) reflects individual mechanical activation levels at lower contraction intensity.
\end{abstract}

Keywords: mechanomyogram, twitch interpolation, $\mathrm{MMG}$, medial gastrocnemius muscle, soleus muscle

[International Journal of Sport and Health Science Vol.5, 63-70, 2007]

\section{Introduction}

When an electrical stimulus was delivered to nerve or muscle during voluntary contraction (interpolated twitch technique), a twitch-like increment force superimposed onto voluntary contraction force appeared. This twitch force superimposed onto voluntary contraction force (superimposed twitch) decreases with an increase of voluntary contraction force. If every muscle fiber reaches peak activation during maximal voluntary contractions, no superimposed twitch amplitude is elicited (Belanger \& McComas, 1981; Merton, 1954). A continuous decrease of superimposed twitch amplitude with an increase of voluntary contraction force was firstly reported by Denny-Brown (1928), followed by the report by Merton (1954), describing that there was a negative relationship between voluntary contraction force and superimposed twitch amplitude. Later, calculation of a voluntary activation ratio was designed by proportion of twitch amplitude evoked at rest and superimposed twitch amplitude evoked during voluntary contractions, which has been employed in a great deal of research as an index to estimate levels of muscle activation (Jakobi \& Rice, 2002; Boerio, et al., 2005; Herbert \& Gandevia, 1999; Shima, et al., 2002). However, a twitch force is thought to be the final output of muscle contractions, and levels of synergistic muscle activation are hardly evaluated individually from superimposed twitch amplitude in such activations as those in which more than one muscle is synergistically activated.

Mechanomyogram (MMG) is a noninvasive 
technique to estimate muscle activation level (Akataki, et al., 2004; Bolton, et al., 1989; Maton, et al., 1990; Orizio, et al., 1990; Shima, et al., 2007). MMG measures pressure waves (micro oscillation) generated from lateral expansion of muscle fiber when contracting (Orizio, 1993). Thus, MMG reflects mechanical activation of muscles (Gordon \& Holbourn, 1948; Yoshitake, et al., 2002; Yoshitake, et al., 2005). In a report by Chen, et al., (1997), which employed a technique combining MMG and interpolated twitch technique, it was confirmed that MMG amplitude superimposed (superimposed MMG amplitude) during voluntary contractions in the adductor pollicis muscle decreased with an increase of voluntary contraction force. Chen, et al., (1997) also reported that superimposed MMG amplitude could be used to assess levels of muscle activation. However, there are few reports on techniques combining MMG and interpolated twitch technique. To our knowledge, there are few studies which simultaneously measure superimposed MMG amplitude of synergistic muscles in activations synergistically involving more than one muscle like plantar flexion.

If superimposed MMG amplitude elicited during voluntary contractions can assess levels of muscle activation, superimposed MMG amplitude can individually evaluate activation levels of synergistic muscles, in which more than one muscle synergistically activates muscle contractions. This study examined changes of mechanical activation levels of medial gastrocnemius muscle (MG, hereafter) and soleus muscle (SOL, hereafter) in different contraction intensities based on information from superimposed MMG amplitude.

\section{Method}

\subsection{Subjects}

Thirteen men, aged $23.8 \pm 3.8$ years; height, 172.3 $\pm 3.9 \mathrm{~cm}$, and weight, $69.8 \pm 5.6 \mathrm{~kg}$, (means $\pm \mathrm{SD}$ ), volunteered for this study. All subjects were tested in the right lower extremity. Prior to the experiment, all subjects received a full explanation of the purpose of the experiment based on the descriptions approved by the Ethics Committee of Osaka University of Health and Sport Sciences, then their written agreement of participation was obtained.

\subsection{Procedure}

We tested MG and SOL of the subjects. Isometric plantar flexion force was measured by a dynamometer (KIN-COM 500H, Chattecx, USA). Each subject was seated with the hip flexed to $80^{\circ}$, knee joint at $0^{\circ}$ (straight knee) and the ankle joint dorsiflexed to $5^{\circ}$. A padded belt was strapped to the dynamometer to support and keep the limb position unchanged during testing. First, maximal voluntary torque (MVC) of isometric plantar flexion was measured; then, the subject performed plantar flexion at the requested force levels of $20 \%, 40 \%$, $60 \%, 80 \%$, and $100 \% \mathrm{MVC}$ in random order. Each trial was intermitted with resting for more than $15 \mathrm{~min}$ to exclude influence of post-activation potentiation (on evoked twitch torque and MMG amplitude) (Vandervoort, et al., 1983; Shima, et al., 2006). Plantar flexion force was displayed by the dynamometer's monitor, which display was used as a visual feedback to let the subjects maintain constant muscle force. When muscle force became constant in about $5 \mathrm{~s}$ from muscle activation, an electrical stimulation was applied to the posterior tibial nerve to record superimposed twitch amplitude and superimposed MMG amplitude from MG and SOL. The superimposed twitch amplitude and superimposed MMG amplitude were measured once at each force level.

To evoke twitches of plantar flexors and induce MMG amplitude in MG and SOL, the posterior tibial nerve was stimulated percutaneously with a surface electrode (cathode, $6 \mathrm{~mm}$ in diameter) in the popliteal fossa. The anode $\left(4 \times 3 \mathrm{~cm}^{2}\right.$ stainless plate) was positioned on the lower one fourth of the front of the thigh. A Square-wave for a duration of $1 \mathrm{~ms}$ was used for electrical stimulation (S88k, Grass Telefactor, USA). The stimulus intensity was incremented until it reached a peak twitch force. At this point, maximal twitch force was checked in each subject and its $120 \%$ intensity was determined for the settings. MMG amplitude was measured using a uniaxial accelerometer (MP110-10-101, Medisens INS, Japan). The accelerometer was attached to the center of the muscle belly by double-faced adhesive tape. We magnified MMG amplitude five times by an amplifier and derived the amplitude in a frequency band of $0.1 \mathrm{~Hz}$ to $1 \mathrm{kHz}$. Twitch and MMG amplitude were taken into the computer using an $\mathrm{AD}$ converter (PowerLab 8sp, ADInstrment, Australia) in 
A

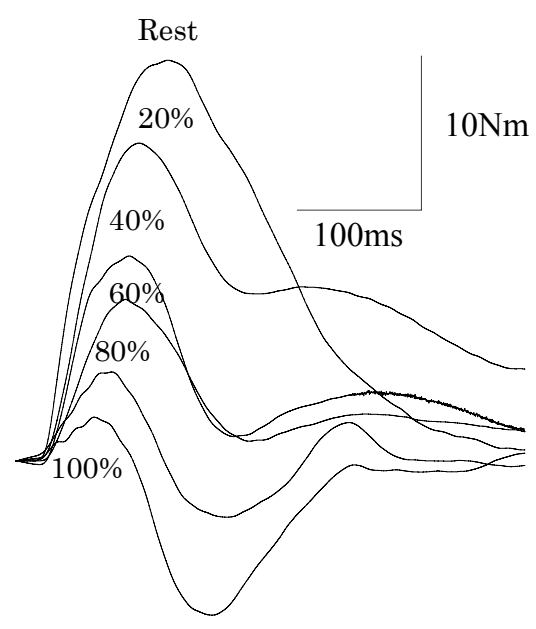

B

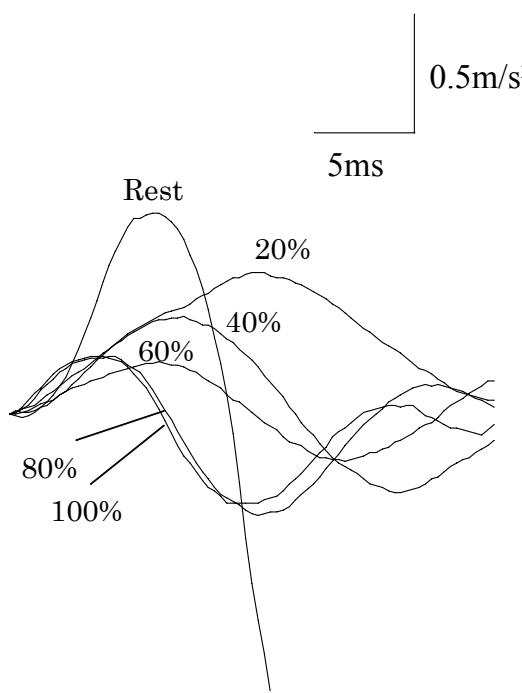

$\mathrm{C}$

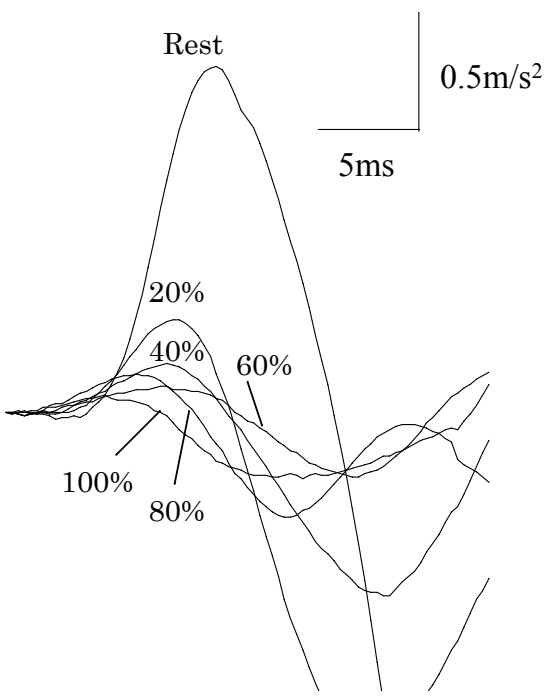

Figure 1 Typical data of superimposed twitch (A) and superimposed mechanomyogram of medial gastrocemius muscle (B) and soleus muscle $(\mathrm{C})$ recordings from a representative subject at rest, $20 \%, 40 \%, 60 \%, 80 \%$, and $100 \%$ of maximal voluntary contraction.

a sampling frequency of $4 \mathrm{kHz}$, and were analyzed by analysis software (chart V5.0.1, ADInstrument, Australia).

\subsection{Data Processing}

Electrically stimulated superimposed twitch amplitude developed at each \%MVC was measured. The peak acceleration of the twitch torque development $\left(\mathrm{d}^{2} T / \mathrm{d} t^{2}\right)$ was measured. The $\mathrm{d}^{2} T / \mathrm{d} t^{2}$ was calculated from the second derivative of the superimposed twitch at each \%MVC. For MMG, amplitude values were measured from application of electrical stimulation to the first peak.

\subsection{Statistical Analysis}

Each measuring value of the 13 subjects was obtained from mean \pm standard deviation (SD). To investigate the effect of force level on superimposed twitch amplitude and $\mathrm{d}^{2} T / \mathrm{d} t^{2}$, repeated one-way ANOVA was used. Then, Tukey's multiple comparison test was employed for the significant items (rest vs. $20 \%, 20 \%$ vs. $40 \%, 40 \%$ vs. $60 \%$, $60 \%$ vs. $80 \%, 80 \%$ vs. $100 \%$ ). Repeated two-way ANOVA (contraction intensity $\times$ muscle) was used to determine the pattern of difference in changes of superimposed MMG amplitude in MG and SOL.
Then, Tukey's multiple comparison test was used for significant items obtained in this test. Linear regression analysis and curve estimation were used to determine the most appropriate regression line and curve for correlations between superimposed twitch amplitude and superimposed MMG amplitude of MG and SOL, and between $\mathrm{d}^{2} T / \mathrm{d} t^{2}$ and superimposed MMG amplitude of MG and SOL. The significant level of all tests was less than 5\%. SPSS software (SPSS 11.0, SPSS, USA) was used for these statistical analyses.

\section{Results}

Figure 1 shows the representative examples of changes of electrically stimulated superimposed twitch amplitude (Figure 1A) and superimposed MMG amplitude in MG (Figure 1B) and SOL (Figure 1C) evoked at rest, and at 20\%, 40\%, 60\%, $80 \%, 100 \%$ MVC. Figure 2 shows the results normalized at rest from the obtained values at each $\% \mathrm{MVC}$ of superimposed twitch amplitude, $\mathrm{d}^{2} T / \mathrm{d} t^{2}$, and superimposed MMG amplitude in MG and SOL. Although the superimposed twitch amplitude (Figure 2A) significantly decreased until $80 \% \mathrm{MVC}$ (rest vs. $20 \%, 20 \%$ vs. $40 \%, 40 \%$ vs. $60 \%, 60 \%$ vs. $80 \%$ ), it did not observe any significant changes after that ( $80 \%$ vs. $100 \%)$. The $\mathrm{d}^{2} T / \mathrm{d} t^{2}$ (Figure 2B) 

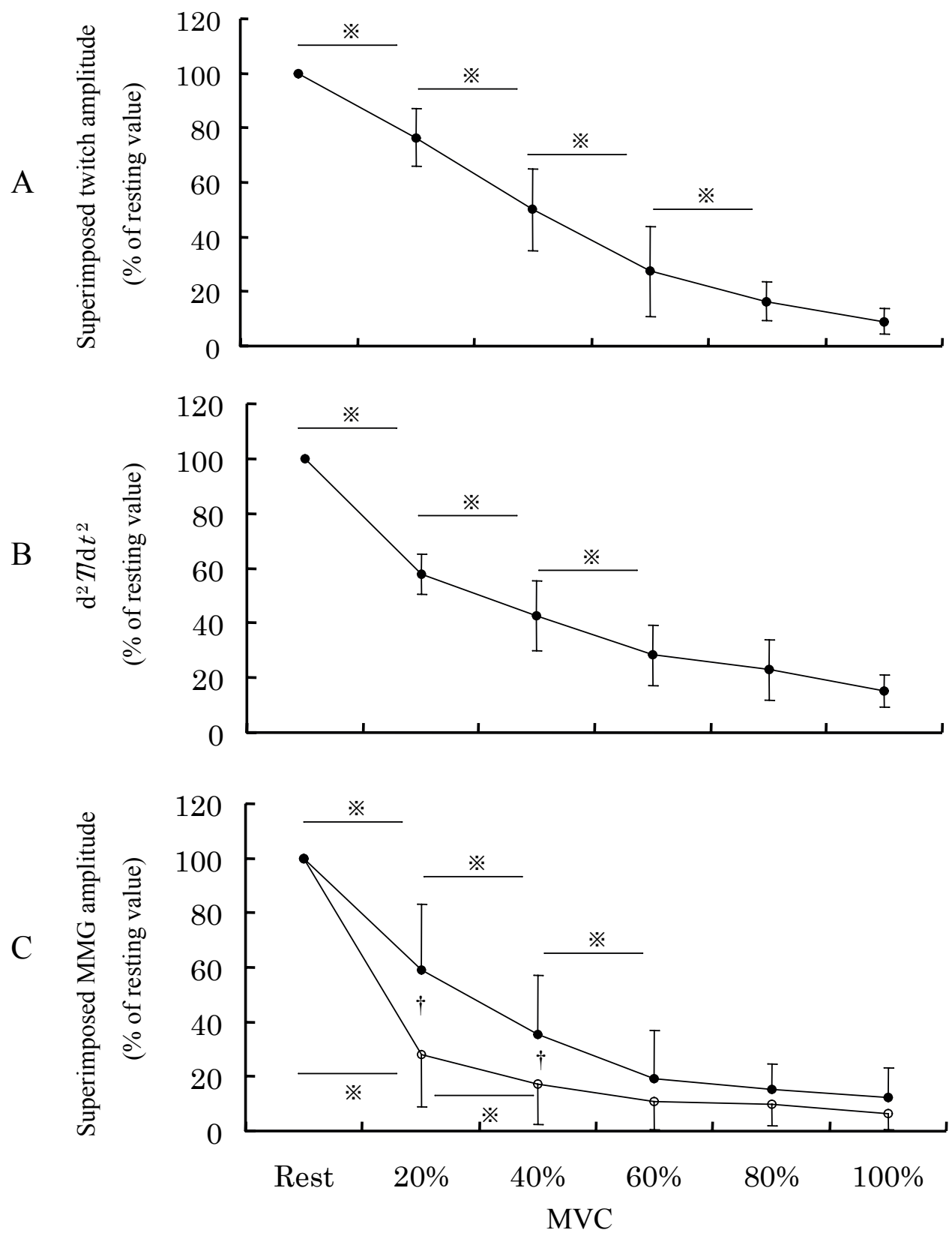

Figure 2 Amplitude of the superimposed twitch (A), the peak acceleration of twitch torque development $\left(\mathrm{d}^{2} T / \mathrm{d} t^{2}\right)(\mathrm{B})$, amplitude of the superimposed mechanomyogram (MMG) (C) of the medial gastrocnemius muscle (MG) (close) and soleus muscle (SOL) (open) at rest, 20\%, 40\%,60\%,80\%, and 100\% of maximal voluntary contraction (MVC). All values are means (SD) for 13 subjects.

※ Significant difference between rest vs. $20 \%, 20 \%$ vs. $40 \%, 40 \%$ vs. $60 \%, 60 \%$ vs. $80 \%$, and $80 \%$ vs. $100 \%$ MVC at $p<0.05$. $\dagger$ Significant difference between MG and SOL at $\mathrm{p}<0.05$.

recognized significant decrease until $60 \% \mathrm{MVC}$ (rest vs. $20 \%, 20 \%$ vs. $40 \%, 40 \%$ vs. $60 \%$ ) but did not show any significant changes after that $(60 \%$ vs. $80 \%, 80 \%$ vs. $100 \%$ ). In the two-way ANOVA of superimposed MMG amplitude changes in MG and SOL (Figure 2C), contraction intensity factors had significant interaction with muscle factors. In the multiple comparison test, the superimposed MMG amplitude recorded from MG observed significant decrease until $60 \% \mathrm{MVC}$ (rest vs. $20 \%, 20 \%$ vs. $40 \%, 40 \%$ vs. $60 \%$ ) but after that there were no significant changes $(60 \%$ vs. $80 \%, 80 \%$ vs. $100 \%)$. The superimposed MMG derived from SOL had a significant decrease until 40\%MVC (rest vs. $20 \%$, $20 \%$ vs. $40 \%$ ) but did not have significant changes after that $(40 \%$ vs. $60 \%, 60 \%$ vs. $80 \%, 80 \%$ vs. $100 \%)$. In comparison with the superimposed MMG amplitude between MG and SOL, 20\% and 
A
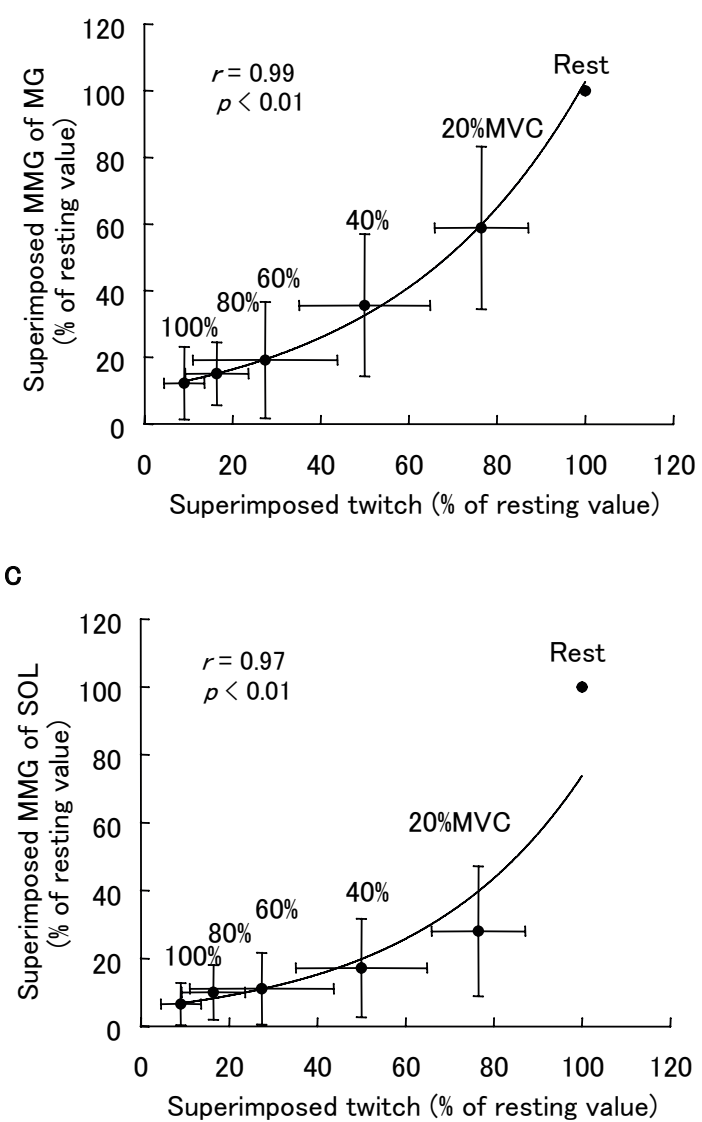

B

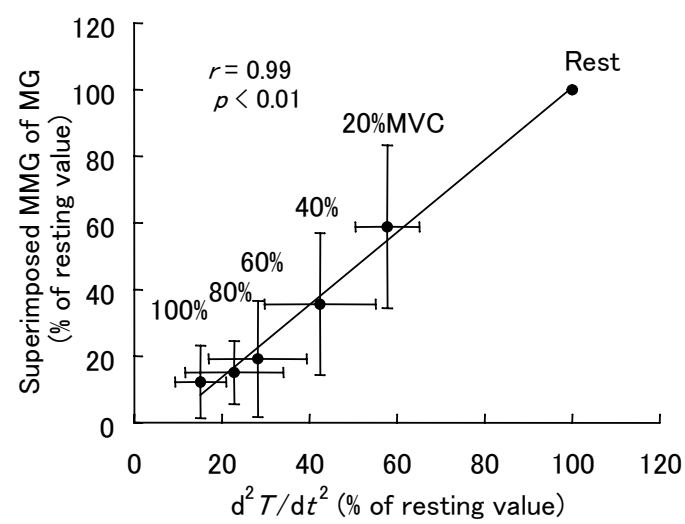

D

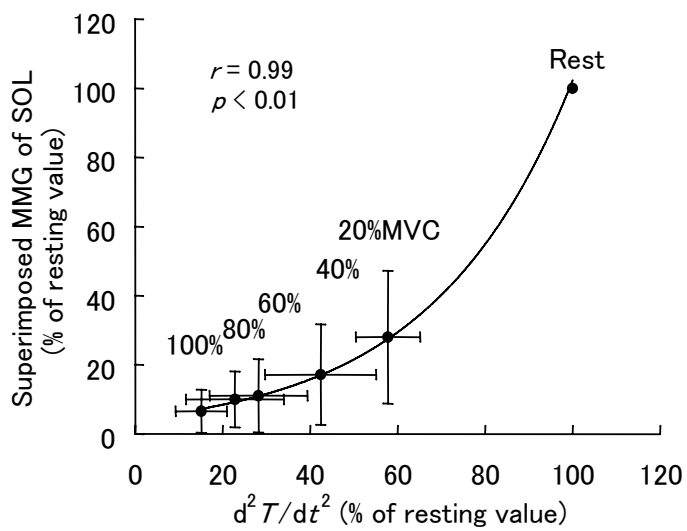

Figure 3 Relationships between the superimposed mechanomyogram (MMG) amplitude of the medial gastrocnemius muscle (MG) and the superimposed twitch amplitude (A), and the peak acceleration of twitch torque development $\left(\mathrm{d}^{2} T / \mathrm{d} t^{2}\right)(\mathrm{B})$. Relationships between the superimposed MMG amplitude of the soleus muscle (SOL) and the superimposed twitch amplitude (C), and $\mathrm{d}^{2} T / \mathrm{d} t^{2}$ (D). Each point corresponds to the mean value (SD) obtained for 13 subjects. Lines of best fit are shown as solid lines.

40\%MVC in MG recognized significantly higher values than those in SOL.

Figure 3 shows the correlations between superimposed twitch amplitude and superimposed MMG amplitude (A) in MG, between $\mathrm{d}^{2} T / \mathrm{d} t^{2}$ and superimposed $M M G$ amplitude (B) in $M G$, superimposed twitch amplitude and superimposed MMG amplitude (C) in SOL, and $\mathrm{d}^{2} T / \mathrm{d} t^{2}$ and superimposed MMG amplitude (D) in SOL. The values are the means of normalized relative values using the values evoked at rest. Strong correlations were observed in exponential regression between superimposed twitch amplitude and superimposed MMG amplitude (A) of MG, superimposed twitch amplitude and superimposed MMG amplitude (C) of SOL, and $\mathrm{d}^{2} T / \mathrm{d} t^{2}$ and superimposed MMG amplitude (D) of SOL (A: $r=0.99, p<0.01, \mathrm{C}: r=0.97, p<$ 0.01, D: $r=0.99, p<0.01)$. Strong correlations were observed in linear regression between $\mathrm{d}^{2} T / \mathrm{d} t^{2}$ and superimposed MMG amplitude (B) of MG (B: $r=$
$0.99, p<0.01)$.

\section{Discussion}

The present study clarified the following points:

1) Superimposed MMG amplitude is related to superimposed twitch amplitude and $\mathrm{d}^{2} T / \mathrm{d} t^{2}$.

2) Superimposed MMG amplitude had different decreasing pattern between MG and SOL with an increase of voluntary contraction force. At $20 \%$ and $40 \% \mathrm{MVC}$, superimposed $\mathrm{MMG}$ amplitude of MG showed significantly higher values than those of SOL.

\subsection{Changes of Superimposed Twitch Amplitude with Increase of Voluntary Contraction Force}

The present study, like previous studies (Belanger \& McComas, 1981; Merton, 1954), observed that superimposed twitch amplitude 
decreased with an increase of voluntary contraction force. No significant change was found even with a decreasing tendency from $80 \%$ to $100 \%$ MVC. In similar reports on triceps surae muscle, superimposed twitch amplitude changed less in the level of contraction in high intensities (Belanger \& McComas, 1981; Todd, et al., 2004). The result of the present study is consistent with the results of previous studies. It might be ascribed to the differences in the level of synergistic muscle activation or small changes of muscle length at high contraction intensities (Allen, et al., 1998; Gandevia, 2001).

\subsection{Changes of Superimposed MMG Amplitude with Increase of Voluntary Contraction Force and its Relationship with Superimposed Twitch Amplitude and $\mathrm{d}^{2} \boldsymbol{T} / \mathrm{d} \boldsymbol{t}^{2}$}

In a report which derived superimposed MMG amplitude from adductor pollicis muscle, superimposed MMG amplitude decreased with an increase of voluntary contraction force, and the decreasing rate became lower as the voluntary contraction force became higher (Chen, et al.,1997). The present study as well recognized a decrease of superimposed MMG amplitude of MG and SOL with an increase of voluntary contraction force and lowering of decreasing ratio at high intensity contractions (Figure 2C). This result supports the above-noted report (Chen, et al., 1997). It is assumed, however, that in vivo behavior of muscle contraction during superimposed twitch should be examined in order to clarify causes of decreasing ratio of superimposed MMG amplitude accompanying an increase of voluntary contraction force.

In regard to relationships between superimposed twitch amplitude and superimposed MMG amplitude, there are significant correlations both in $\mathrm{MG}$ and SOL (Figure 3A, C). Inconsistent with the report by Chen, et al., (1997), which recognized a linear relationship in adductor pollicis muscle, the present study showed a curvilinear relationship in $\mathrm{MG}$ and SOL. This difference is possibly associated with the different muscles and different transducer (microphone and accelerometer) used for derivation of MMG.

Further, MMG derived by electric stimulation correlated both with evoked twitch amplitude and its $\mathrm{d}^{2} T / \mathrm{d} t^{2}$ in linear regression (Orizio, et al., 1999; Shima, et al., 2006). The present study also found a significant correlation in linear regression of MG (Figure 3B). This shows that superimposed MMG amplitude reflects changes of superimposed twitch amplitude as well as $\mathrm{d}^{2} T / \mathrm{d} t^{2}$. Since $\mathrm{d}^{2} T / \mathrm{d} t^{2}$ reflects dynamics of calcium released from the muscular endoplasmic reticulum (Takamori, et al., 1971), changes of superimposed MMG amplitude may possibly reflect excitation-contraction coupling (E-C coupling). In the meantime, superimposed MMG amplitude of SOL showed a curvilinear relationship with $\mathrm{d}^{2} T / \mathrm{d} t^{2}$, which was different from MG (Figure 3D). This probably reflects differences in ratio of muscle fiber composition between these muscles.

\subsection{Difference in Superimposed MMG Amplitude between MG and SOL}

The present study showed interaction between contraction intensity factors and muscle factors in superimposed MMG amplitude (Figure 2C). The result showed differences in the change in pattern of superimposed MMG amplitude between MG and SOL with an increase of voluntary contraction force. In a report by Yoshitake \& Moritani (1999), which measured MMG of MG and SOL during voluntary contractions, there were differences in the MMG root mean square (MMGrms) between MG and SOL with an increase of voluntary contraction force. Yoshitake \& Moritani (1999) described that the MMGrms was different between MG and SOL because the relative contribution to development of output force was different between the muscles. In our comparison of superimposed MMG amplitude between MG and SOL at each $\% \mathrm{MVC}$, the superimposed MMG amplitude of $\mathrm{MG}$ at $20 \%$ and $40 \% \mathrm{MVC}$ showed comparatively higher significant values than those of SOL. MMG signal reflects mechanical activity at muscle contraction (Gordon \& Holbourn, 1948; Orizio, 1993; Yoshitake, et al., 2005). Motor unit without activation states produces superimposed twitch force during electrical stimulus onto submaximal voluntary contraction (Belanger \& McComas, 1981; Shield \& Zhou, 2004). As previously mentioned, if superimposed MMG amplitude reflects not only superimposed twitch amplitude but $\mathrm{d}^{2} T / \mathrm{d} t^{2}$, it can be interpreted that superimposed MMG amplitude indicates changes of 
muscle mechanical activity (including $\mathrm{d}^{2} T / \mathrm{d} t^{2}$ ) when non-recruited motor units are evoked by electrical stimulation during voluntary contraction. These suggest that differences in the decreasing pattern of superimposed MMG amplitude between MG and SOL with an increase of voluntary contraction force might be caused by different mechanical activity levels between MG and SOL. For instance, superimposed MMG amplitude of MG showed higher values than SOL at $20 \%$ and $40 \%$ MVC because the mechanical activity level of MG might be lower than SOL at $20 \%$ and $40 \% \mathrm{MVC}$. These results suggest that superimposed MMG amplitude synchronously recorded from MG and SOL during voluntary contraction in low intensities reflects mechanical activity levels of these muscles individually.

\section{Conclusion}

MG and SOL had different patterns in the level of mechanical activity in superimposed MMG amplitude with an increase of voluntary contraction force. The difference in the level of mechanical activity between both muscles was observed during voluntary contraction in low intensities. Accordingly, it is suggested that superimposed MMG amplitude during voluntary contraction of plantar flexion in low intensities can evaluate differences in mechanical activity levels between MG and SOL.

\section{Acknowledgements}

This research was greatly supported by Yukiko Miki, Hitomi Urano, and Akinobu Tsumura of Our Research Laboratory of the Graduate School of Osaka University of Health and Sport Sciences. In writing the paper, Soichi Ando (Special Researcher of the Japan Society of the Promotion of Science, Osaka University of Health and Sport Sciences) supported me with valuable advice. I would like to express my gratitude here. I also appreciate the cooperation of the subjects in the study.

\section{References}

Akataki, K., Mita, K., \& Watakabe, M. (2004). Electromyographic and mechanomyograhic estimation of motor unit activation strategy in voluntary force production. Electromyography and Clinical Neurophysiology, 44: 489-496.

Allen, G. M., McMenzie, D.K., \& Gandevia, S.C. (1998). Twitch interpolation of the elbow flexor muscles at high forces. Muscle \& Nerve, 21: 318-328.

Belanger, A.Y., \& McComas, A.J. (1981). Extent of motor unit activation during effort. Journal of Applied Physiology, 51: 1131-1135.

Boerio, D., Jubeau, M., Zory, R., \& Maffiuletti, N.A. (2005). Central and peripheral fatigue after electrostimulation-indu ced resistance exercise. Medicine and Science in Sports and
Exercise, 37: 973-978.

Bolton, C.F., Parkes, A., Thompson, T.R., Clark, M.R., \& Sterne, C.J. (1989). Recording sound from human skeletal muscle: Technical and physiological aspects. Muscle \& Nerve, 12: 126-134.

Chen, D., Durand, L.G., \& Bellemare, F. (1997). Time and frequency domain analysis of acoustic signals from a human muscle. Muscle \& Nerve, 20: 991-1001.

Denny-Brown, D. (1928). On inhibition as a reflex accompaniment of the tendon jerk and of other forms of active muscular response. Proceedings of the Royal Society of London. Series B, Containing Papers of a Biological Character, 103: 321-336.

Gandevia, S.C. (2001). Spinal and supraspinal factors in human muscle fatigue. Physiological Reviews, 81: 1725-1789.

Gordon, G., \& Holbourn, A.H.S. (1948). The sounds from single motor units in a contracting muscle. Journal of Physiology, 107: 456-464.

Herbert, R.D., \& Gandevia, S.C. (1999). Twitch interpolation in human muscle: Mechanisms and implications for measurement of voluntary activation. Journal of Neurophysiology, 82: 2271-2283.

Jakobi, J.M., \& Rice, C.L. (2002). Voluntary muscle activation varies with age and muscle group. Journal of Applied Physiology, 93: 457-462.

Maton, B., Petitjean, M., \& Conckaert, J.C. (1990). Phonomyogram and electromyogram relationships with isometric force reinvestigated in man. European Journal of Applied Physiology, 60: 194-201.

Merton, P.A. (1954). Voluntary strength and fatigue. Journal of Physiology, 123: 553-564.

Orizio, C., Diemont, B., Esposito, F., Alfonsi, E., Parrinello, G., Moglia, A., \& Veicsteinas, A. (1999). Surface mechanomyogram reflects the changes in the mechanical properties of muscle at fatigue. European Journal of Applied Physiology and Occupational Physiology, 80: 276-284.

Orizio, C., Perini, R., Biemont, B., Figini, M.M., \& Veicsteinas, A. (1990). Spectral analysis of muscular sound during isometric contraction of biceps brachii. Journal of Applied Physiology, 68: 508-512.

Orizio, C. (1993). Muscle sound: Bases for the introduction of a mechanomyographic signal in muscle studies. Critical Reviews in Biomedical Engineering, 21: 201-243.

Shield, A., \& Zhou, S. (2004). Assessing voluntary muscle activation with the twitch interpolation technique. Sports Medicine, 34: 253-267.

Shima, N., Ishida, K., Katayama, K., Morotome, Y., Sato, Y., \& Miyamura, M. (2002). Cross education of muscular strength during unilateral resistance training and detraining. European Journal of Applied Physiology, 86: 287-294.

Shima, N., Rice, C.L., Ota, Y., \& Yabe, K. (2006). The effect of postactivation potensiation on the mechanomyogram. European Journal of Applied Physiology, 96: 17-23.

Shima, N., McNeil, C. J., \& Rice, C. L. (2007). Mechanomyographic and electromyographic responses to stimulated and voluntary contractions in the dorsiflexors of young and old men. Muscle \& Nerve, 35: 371-378.

Takamori, M., Gutmann, L., \& Shane, S. R. (1971). Contractile properties of human skeletal muscle. Normal and thyroid disease. Archives of Neurology, 25: 535-546.

Todd, G., Gorman, R.B., \& Gandevia, S.C. (2004). Measurement and reproducibility of strength and voluntary 
activation of lower-limb muscles. Muscle \& Nerve, 29: 834-842.

Vandervoort, A.A., Quinlan, J., \& McComas, A.J. (1983). Twitch potentiation after voluntary contraction. Experimental Neurology, 81: 141-152.

Yoshitake, Y., Kawakami, Y., Kanehisa, H., \& Fukunaga, T. (2005). Surface mechanomyogram reflects length changes in fascicles of human skeletal muscles. International Journal of Sport and Health Science, 3: 280-285.

Yoshitake, Y., \& Moritani, T. (1999). The muscle sound properties of different muscle fiber types during voluntary and electrically induced contractions. Journal of Electromyography and Kinesiology, 9: 209-217.

Yoshitake, Y., Shinohara, M., Ue, H., \& Moritani, T. (2002). Characteristics of surface mechanomyogram are dependent on development of fusion of motor units in humans. Journal of Applied Physiology, 93: 1744-1752.

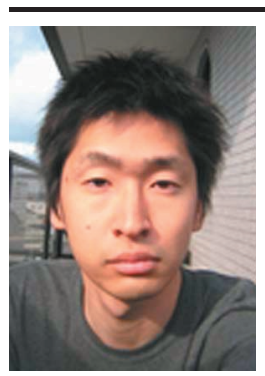

Name:

Yoichi Ohta

\section{Affiliation:}

Department of Sport and Exercise Sciences, Graduate School of Sport Sciences, Osaka University of Health and Sport Sciences

\section{Address:}

1-1 Asashirodai, Kumatori, Sennan-gun, Osaka 590-0496 Japan Brief Biographical History:

2005- Received a Master's degree in Sport Science from Osaka University of Health and Sport Sciences

2005- Doctoral Program, Graduate School of Sport Sciences, Osaka University of Health and Sport Sciences

Main Works:

- "The effect of postactivation potensiation on the mechanomyogram." European Journal of Applied Physiology, 96: 17-23, 2006

Membership in Learned Societies:

- Japan Society of Physical Education, Health and Sport Sciences

- Japanese Society of Physical Fitness and Sports Medicine

- Japanese Society of Biomechanics

- Japan Society of Exercise and Sports Physiology 